with respect to the number of patients with kinesiophobia and to mean scores of pain intensity, fatigue, $H A Q$ and $B D I(p<0,05-<0,001)$. Patients with RA had higher rates of kinesiophobia than patients with $A S$ and healthy controls $(p=0.001$, $\mathrm{p}=0,001$ ). Patients with RA had worser scores than patients with AS and healthy controls. Patients with AS had worser scores than healthy controls. In patients with RA and AS, kinesiophobia is associated with pain severity, fatigue, emotional status and QoL.

Conclusions: In our study, patients with RA and AS had higher rates of kinesiophobia. We found that kinesiophobia was related with pain severity, fatigue, depression, disease activity and QoL of the patients. The QoL can be improved through controlling kinesiophobia by reducing pain, depression and fatigue.

Disclosure of Interest: None declared

DOI: 10.1136/annrheumdis-2017-eular.6163

\section{AB0256 SERUM AND SYNOVIAL KYNURENIC ACID CONCENTRATION AND ITS CORRELATION WITH DISEASE ACTIVITY IN PATIENTS WITH RHEUMATOID ARTHRITIS: CLINICAL AND ULTRASONOGRAPHIC STUDY}

F. Elshabacy ${ }^{1}$, T. Fathy Mohammed ${ }^{1}$, M.A. Mortada ${ }^{2}$, H.A. EL-Saadany ${ }^{3}$, S.M. Farouk ${ }^{4}$, E.R. Amer ${ }^{4} .{ }^{1}$ Rheumatology, Benha Teaching Hospital, Benha; ${ }^{2}$ Rheumatology \& Rehabilitation, Zagazig University, Zagazig; ${ }^{3}$ internal medicine and Rheumatology, Military Medical Academy, Cairo; ${ }^{4}$ Clinical Pathology, Benha Teaching Hospital, Benha, Egypt

Background: Rheumatoid arthritis (RA) is a chronic inflammatory disease. kynurenic acid has anti-inflammatory effects, because it is the most important agonist of the orphan G-protein-coupled receptor (GPR35) which expressed on various types of cells associated with the immune system. Stimulation of these receptors by kynurenic acid lead to reduction in the synthesis of proinflammatory cytokines, nitric oxide, and reactive oxygen species (1).

Objectives: Detection and quantification of kynurenic acid in serum and synovial fluid obtained from the affected joints in patients with rheumatoid arthritis and its relation to different clinical aspects of disease activity and signs of synovitis and synovial hyperplasia detected by musculoskeletal diagnostic ultrasound.

Methods: Thirty RA patients diagnosed according to ACR and EULAR revised criteria and thirty patients of idiopathic knee osteoarthritis as a control group were enrolled in the study. These patients were collected from outpatient clinic of rheumatology department Benha Teaching Hospital. Kynurenic acid was assessed in Serum samples from all patients and controls coupled synovial fluid samples aspirated from knee joint of all RA patients and fourteen OA patients after musculoskeletal ultrasonographic examination of these joints.

Results: Serum and synovial level of kynurenic acid was assessed in the studied groups. Comparison between RA and OA patients as regard serum kynurenic acid showed no differences where it's level was $29.80 \pm 13.86 \mathrm{pg} / \mathrm{ml}$ in $R A$ versus $30.98 \pm 11.03 \mathrm{pg} / \mathrm{ml}$ in $O A$ patients), while synovial kynurenic acid was significantly lower in RA $16.38 \pm 6.45 \mathrm{pg} / \mathrm{ml}$ than in OA patients $26.22 \pm 2.99 \mathrm{pg} / \mathrm{ml}(p<0.001)$. kynurenic acid was significantly lower in synovial fluid $(16.38 \pm 6.45 \mathrm{pg} / \mathrm{ml})$ than in serum $(29.80 \pm 13.86 \mathrm{pg} / \mathrm{ml})$ in RA group of patients $(p<0.001)$. Comparison among different grades of synovitis detected by grey scale U/S and by Doppler signals in RA patients as regard synovial kynurenic acid showed that it was significantly lower in higher grades of synovitis $(P<0.001)$. Synovial kynurenic acid level was negatively correlated with grades of synovitis and Doppler signals $(p<0.001)$

Conclusions: The negative correlation between Kynurenic acid concentration in the synovial fluid and both the synovial thickness detected by ultrasonography and the hyperaemia of synovial tissues as represented by the Doppler activity, may support its use as a local marker of the two faces of rheumatoid arthritis (chronicity and activity) at the joint level.

To the best of our knowledge, this is the first study that gives correlation between the serum and synovial levels of kynurenic acid concentrations and the grade of synovitis detected by grey scale and Doppler ultrasonography.

\section{References:}

[1] Tistzlavicz Z, Nemeth B, Fulop F et al (2011). Naunyn Schmiedebregs Arch Phrmacol; 383(5):447-55.

Disclosure of Interest: None declared

DOI: 10.1136/annrheumdis-2017-eular.2198

\section{AB0257 SCREENING OF DIFFERENTIALLY EXPRESSED SERUM PROTEINS FOR RHEUMATOID ARTHRITIS BY SURFACE-ENHANCED LASER DESORPTION/INIONATION-TIME OF FLIGHT-MASS SPECTRA}

N. Liang, L. Zhang, K. Xu. Shanxi academy of medical sciences Shanxi dayi hospital, TaiYuan, China

Background: RA is a chronic inflammatory rheumatic disease, and early diagnosis and treatment can improve prognosis ${ }^{1}$.Surface-enhanced laser parsing ionization/time of flight mass spectrometry (SELDI-TOF-MS) combining a protein chip and mass spectrometry technology own the advantages of low dosage of samples, direct point sample detection and high sensitivity.

Objectives: SELDI used to profile and compare the proteomes in serum samples of RA patients including complicated with Sjogren's syndrome (SS), interstitial lung disease (ILD). Using Biomarker Wizard software and Biomarker Pattern Software established diagnostic model, and calculate sensitivity and specificity,which can simplify clinical procedures, save medical costs and explore the pathogenesis.

Methods: Using the Biomarker Wizard and Biomarker Pattern software to establish the diagnosis prediction model to predict RA disease progression between the following groups, including simple RA patients $(n=44)$ and RA-SS patients $(n=18)$, RA patients $(n=44)$ and RA-ILD $(n=22)$, RA patients $(n=44)$ and RA-ONFH $(n=6)$. Also 96 RA patients and 77 healthy control, which were randomly allocated to the training set ( 83 RA patients and 56 healthy controls) and test set (14 RA patients and 20 healthy controls)to develop and verify a pattern by means of decision treealgorithm.

Using the Biomarker Wizard and Biomarker Pattern software to establish the diagnosis prediction model to predict RA disease progression between the following groups, including simple RA patients $(n=44)$ and RA-SS patients $(n=18)$, RA patients $(n=44)$ and RA-ILD $(n=22)$. Also 96 RA patients and 77 healthy control, which were randomly allocated to the training set ( 83 RA patients and 56 healthy controls) and test set (14 RA patients and 20 healthy controls)to develop and verify a pattern by means of decision treealgorithm.

Results: 1. Comparison of RA patients and healthy controls: there are 22 up-regulated expression in RA, 36 down-regulated. The diagnostic model of

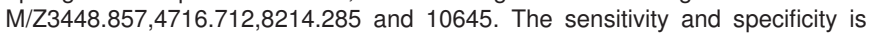
$91.566 \%$ and $92.857 \%$, the area under the ROC curve was 0.937 , to verify the diagnosis model, we get a sensitivity of $100 \%$ and a specificity of $95 \%$. 2 . Comparison of the simple RA and RA -ILD. The diagnostic model of M/Z10645.1, $\mathrm{M} / \mathrm{Z12595.86}$. The sensitivity and specificity is $86.4 \%$ and $84.1 \%$ and the area under the ROC curve was 0.856.3. Comparison of the simple RA and RA -SS: The diagnostic model of $\mathrm{M} / \mathrm{Z6635.623}, \mathrm{M} / \mathrm{Z33897.72}$. The sensitivity and specificity is $77.8 \%$ and $79.5 \%$ and the area under the ROC curve was 0.794 .

Conclusions: The serum protein fingerprinting by SELDI-TOF-MS could identify new biomarkers in RA. The biomarkers may play an important role in pathogenesis of RA. We could diagnose RA in early stage, predict disease progression and determine disease activity by these biomarkers.

References:

[1] Vincent Goëb1, Marlène Thomas-L'Otellier2,et al. Candidate autoantigens identified by mass spectrometry in early rheumatoid arthritis are chaperones and citrullinated glycolytic enzymes. Arthritis Research \& Therapy, 2009, $11:$ R38.

[2] Jr GW, Cazares LH, Leung SM, Nasim S, Adam BL, Yip TT. Protein chip surface enhanced laser desorption/ionization (SELDI) mass spectrometry: a novel protein biochip technology for detection of prostate cancer biomarkers in complex protein mixtures. Prostate Cancer Prostate Dis.1992;2:264-76. Disclosure of Interest: None declared

DOI: 10.1136/annrheumdis-2017-eular.3698

\section{AB0258 AGE AND QUALITY OF LIFE AMONG RHEUMATOID ARTHRITIS PATIENTS TREATED WITH BIOLOGIC AGENTS}

N. Oguro, N. Yajima, Y. Miwa. Rheumatology, Showa University School of Medicine, Nishinakanobu, Shinagawa Ward, Tokyo, Japan

Background: Rheumatoid arthritis (RA) is a common autoimmune disease of unknown etiology which is characterized by symmetric, chronic inflammatory, peripheral polyarthritis. If it is untreated or unresponsive to therapy, inflammation and joint destruction lead to loss of physical function, inability to carry out daily tasks of living.

In addition to problems related to pain and inflammation, patients with RA are also affected by psychological problems such as anxiety and depression.

It has been a while since, the biologic agents have let RA patients to early remmision, and improved their Quality of life (QOL).

A few studies show relevance between age and QOL among RA patients. Lambert et al. found that age was positively correlated with pain, indicating that increasing age made the situation worse.

Objectives: This study aimed to assess the relationship between age and QOL of RA patients who has been treated with biologic agents.

Methods: 149 RA patients who treated with biologic agents at Showa University Hospital, Showa University Northern Yokohama Hospital and Showa University Koto Toyosu Hospital were recruited from 2005 to 2016. This study design was retrospective cohort study. Loss to follow-up was eliminated. The patients were divided into two groups, whose age was 65 years old and over (elderly) and under 65 (adults). The primary outcome was the change of QOL in 6months'. QOL was measure using SF-36, and we use physical component scale (PCS) and mental component scale (MCS). Logistic regression analysis was performed.

Results: Among 149 RA patients, the mean age was 57 years old and $85.9 \%$ was female. 92 out of 149 patients $(61.7 \%)$ were adult group and $57(38.3 \%)$ were elderly group. Adjusted with sex, disease duration, DAS28-ESR, HAQ, and with or without complications which are interstitial lung disease, diabetes mellitus, and chronic kidney disease, there was no significant difference in change of MCS in 6 months'. But those of PCS was significantly higher in adult's group (regression coefficients -7.25 ; $95 \%$ Confidence Interval $(\mathrm{Cl})-11.7$ to $-2.77 ; p=0.0018$ ).

Conclusions: There is a possibility that, younger patient who suffers with RA could achieve better quality of life than those of elderly patients after treatment with biologic agents. 
Table1.

\begin{tabular}{lrlll}
\hline & & \multicolumn{1}{l}{ Total $(\mathrm{n}=149)$} & \multicolumn{1}{l}{ Adults $(\mathrm{n}=92)$} & \multicolumn{1}{l}{ Elderly $(\mathrm{n}=57)$} \\
\hline Age & $\mathrm{mean}(\mathrm{SD})$ & $57(15.4)$ & $47(12.2)$ & $71(5.63)$ \\
Female & $\mathrm{n}(\%)$ & $128(85.9)$ & $77(83.7)$ & $51(89.4)$ \\
Disease duration (year) mean(SD) & $8.4(10.0)$ & $7.1(7.55)$ & $10(12.9)$ \\
DAS28 ESR & mean (SD) & $4.74(1.37)$ & $4.54(1.38)$ & $5.08(1.30)$ \\
HAQ & mean (SD) & $0.61(0.66)$ & $0.49(0.56)$ & $0.78(0.76)$ \\
\hline
\end{tabular}

Table2.

\begin{tabular}{|c|c|c|c|}
\hline & regression coefficient & $95 \% \mathrm{CI}$ & $p$ value \\
\hline Adults & reference & - & - \\
\hline Elderly & -7.24 & -11.7 to -2.7 & 0.0018 \\
\hline
\end{tabular}

Adjuseted with sex, disease duration, DAS28-E

\section{References:}

[1] Izabela Roma (2014) Quality of life and elderly patients with rheumatoid arthritis, Rev Bras Reumatol 2014;54(4):279-286.

[2] ULF Jakobsson (2002) Pain and quality of life among a older people with rheumatoid arthritis and/or osteoarthritis, Journal of clinical Nursing 2002;11;430-443.

Disclosure of Interest: None declared

DOI: 10.1136/annrheumdis-2017-eular.4708

\section{AB0259 PREDICTORS OF HIGH DISEASE ACTIVITY IN A COHORT OF GREEK PATIENTS WITH ACPA POSITIVE EARLY RHEUMATOID ARTHRITIS}

N. Papadopoulos ${ }^{1}$, A. Gkagkalis ${ }^{2}$, E. Konstantinidou ${ }^{3}$, L. Mentesidou ${ }^{1}$, G. Tzatzagou ${ }^{4}$, V. Galanopoulou ${ }^{1} .{ }^{1}$ Rheumatology Unit; ${ }^{2}$ Gastroenterology Unit, Papageorgiou General Hospital; ${ }^{3}$ Molecular Biologist, PRIVATE; ${ }^{4} 1$ st INTERNAL MEDICINE CLINIC, Papageorgiou General Hospital, Thessaloniki, Greece

Background: In Early Rheumatoid Arthritis, the presence of ACPA positivity is associated with increased disease activity.

Objectives: To investigate possible predictors, for high disease activity (DAS-28 $>5.1$ ), in a cohort of Greek patients with ACPA positive early RA.

Methods: From 2000 until December 2016, 156 patients with ACPA positive early RA, were diagnosed and subsequently followed up as outpatients at the Rheumatology Unit of our hospital. Demographic, clinical, laboratory and therapeutic parameters were evaluated during every follow-up. At the end of the study (last visit during 2016), all the above parameters were re-evaluated, considering the high disase activity (DAS-28 $>5.1$ ). The used methods were $\chi^{2}$, ANOVA, Binary Logistic Regression (BLR) and ROC Curve.

Results: From 156 patients, $25 \%$ were males, $37.8 \%$ were current smokers, $76.9 \%$ were RF positive, $34.6 \%$ presenting with extra-articular manifestations and $16 \%$ with anemia of chronic disease (ACD). At the time of diagnosis, Univariate analysis revealed that smokers, RF positivity, high levels of CRP, patients with extra-articular manifestations and those with $\mathrm{ACD}$, corellate statstically significant with high disease activity $(p=0.011, p=0.006, p=0.03, p=0.001$ and $p=0.001$ respectively). Using the BRL we found that the odds ratio for DAS-28 $>5.1$, were for smokers 2.95, for RF positivity 3.397 and for CRP 1.066 respectively. Based on the previous results the severity of disease can be predicted with sensitivity $80 \%$ (area unedr the curve: $\mathrm{p}<0.001, \mathrm{Cl}=0.723-0.878$ ) using the ROC curve.

Conclusions: |High Diseasae Activity in ACPA positive early RA, at diagnosis, is best predicted by smoking, RF positivity and high levels of CRP.

References:

[1] Aletaha D et al, Rheum Dis North Am 2006;32:9-44.

[2] ScottDL et al, Lancet 2020;376:1094-1108.

[3] Papadopoulos N et al, Clin Rev Allerg Immunol 2008;34:11-15.

Disclosure of Interest: None declared

DOI: 10.1136/annrheumdis-2017-eular.6763

\section{AB0260 THE PREDICTORS OF FRAGILITY FRACTURES IN PATIENTS ON AROMATASE INHIBITORS: AN OBSERVATIONAL STUDY}

$\underline{\text { N. Meah }}^{1}$, R. Sinha ${ }^{2}$, M. Bukhari ${ }^{2} .{ }^{1}$ Lancaster Medical School; ${ }^{2}$ Rheumatology, Royal Lancaster Infirmary, Lancaster, United Kingdom

Background: The use of aromatase inhibitors (Als), given after breast cancer, has been associated with a low bone mineral density (BMD) and as a risk factor for fragility fractures. We have reported on the risk factor for low BMD in a previous abstract and it showed that the use of Als significantly reduced lumbar spine BMD and femoral neck BMD (1). The FRAX TM tool uses the femoral neck $\mathrm{BMD}$ to predict fractures on a population basis but ignores the lumbar spine. Some patients who have undergone bilateral hip replacements would not be able to estimate their FRAX TM risk accurately.

Objectives: To determine the predictors of fragility fractures in an observational cohort of female patients on aromatase inhibitors.

Methods: Female patients referred for BMD estimation in a scanner in the North West of England between 2004 and 2014 on aromatase inhibitors were identified from a dual X-ray absorptiometry database. Demographics and other risk factors, as well as fragility fractures, were recorded. Initially, those who had sustained a fracture were compared to those who had not sustained a fracture using chi-squared tests for categorical variables and T-tests for continuous variables. Following that, univariate and multivariate logistic regression models were fitted looking at the predictors of fracture. Variables included age at scan, height, weight, alcohol, smoking, family history, rheumatoid arthritis, secondary osteoporosis as defined by FRAXTM, body mass index and steroid exposure, in addition to BMD in the lumbar spine and femoral neck.

Results: 2029 female patients were scanned in the referral period. The mean age at scan was 66 (SD 10.46). 356/2029 (17.55\%) had sustained a fracture. Results of the univariate analysis are shown in table 1, significant predictors are indicated with an asterisk $\left(^{*}\right)$.

\begin{tabular}{|c|c|c|c|c|c|}
\hline Predictor & All Pts & $\begin{array}{l}\text { Pts with } \\
\text { Fracture }\end{array}$ & $\begin{array}{l}\text { Pts without } \\
\text { Fracture }\end{array}$ & $\mathrm{p}$-value & $\begin{array}{l}\text { Odds ratio } \\
(95 \% \mathrm{Cl})\end{array}$ \\
\hline Age at scan (years) & 35 & 41 & 65.09 & 0.00 & $1.04(1.03,1.05)^{\star}$ \\
\hline Height $(\mathrm{cm})$ & 160.40 & 159.60 & 160.57 & 0.02 & $0.98(0.96,1.00)$ \\
\hline Weight (kg) & 72.38 & 71.35 & 72.60 & 0.15 & $1.00(0.99,1.00)^{*}$ \\
\hline Alcohol & $123(6.06 \%)$ & $22(6.18 \%)$ & 01 (6.04\%) & .92 & $1.03(0.64,1.65)$ \\
\hline moking & $698(34$ & $132(3$ & 56 & 24 & \\
\hline Family $\mathrm{Hx}$ & 247 & & $19 s$ & 1 & \\
\hline RA & 32( & 1 & $\%)$ & 0.04 & 2.17 \\
\hline Secondary o & $183(9.02 \%)$ & $46(12.92 \%)$ & $137(8.19 \%)$ & 0.01 & $1.66(1.17,2.37)^{*}$ \\
\hline $\begin{array}{l}\text { Femoral Neck BMD } \\
\left(\mathrm{g} / \mathrm{cm}^{2}\right)\end{array}$ & 87 & 82 & 0.88 & 0.00 & $0.02(0.01,0.05)^{x}$ \\
\hline & 1.10 & 1.05 & 1.12 & 0.00 & $0.12(0.06,0.24)^{*}$ \\
\hline $\operatorname{BMI}\left(\mathrm{kg} / \mathrm{m}^{2}\right)$ & 28.13 & 28.00 & 28.16 & 0.61 & $0.99(0.97,1.02)$ \\
\hline Steroid & 466 (22.97\%) & 77 (21.63\%) & $389(23.25 \%)$ & 0.51 & $0.91(0.69,1.20)$ \\
\hline
\end{tabular}

In the multivariate model, the variables that predicted fractures in this cohort were age at scan (OR $1.0395 \% \mathrm{Cl} 1.02,1.05$ ), femoral neck BMD (OR $0.7495 \% \mathrm{Cl}$ $0.02,0.29$ ) and lumbar spine BMD (OR $0.3495 \% \mathrm{Cl} 0.14,0.80)$. Using the femoral neck multivariate model resulted in an area under the curve of 0.6720 and using the lumbar spine was 0.6653 .

Conclusions: In the univariate analysis, many risk factors are associated with fractures within this cohort of female patients on aromatase inhibitors but both univariate analysis and multivariate analysis showed that lumbar spine BMD is a good predictor of fractures. This is not included in the FRAX TM tool and would enable fracture risk to be calculated in patients who have undergone hip replacements.

References:

[1] Ann Rheum Dis 2014;73 Suppl3 SAT0472.

Disclosure of Interest: None declared

DOI: 10.1136/annrheumdis-2017-eular.1406

\section{AB0261 CLINICAL AND STRUCTURAL RESPONSES OF PATIENTS WITH ACTIVE RHEUMATOID ARTHRITIS (RA) USING STEP-UP DOSAGES OF TOFACITINIB IN A TREAT TO TARGET APPROACH}

${ }_{\text {N.B. Gaylis }}{ }^{1}$, J. Sagliani ${ }^{1}$, S. Needell ${ }^{2} .{ }^{1}$ Arthritis and Rheumatic Disease Specialties, Aventura; ${ }^{2}$ Boca Radiology, Boca Raton, United States

Background: Tofacitinib has been shown to reduce the clinical signs and symptoms of some RA patients at an approved dose of $5 \mathrm{mg}$ bid. Studies report that $10 \mathrm{mg}$ bid is an effective dose. This is the first community practice trial to measure the clinical and structural benefits of stepping up the initial dose of $5 \mathrm{mg}$ bid in non-responders to $10 \mathrm{mg}$ bid in order to achieve a clinical response using a treat to target approach.

Objectives: This study evaluates the optimal dose of tofacitinib (5 mg bid VS 10 $\mathrm{mg}$ bid) needed to reach treatment target in a cohort of patients with active RA while comparing the corresponding structural findings measured by low field MRI. Methods: 20 RA patients who were unresponsive to either methotrexate (10-25 mg weekly) or MTX plus up to 2 prior biologics with synovitis, osteitis or erosions on Baseline MRI (Esaote 0.3T) were treated with $5 \mathrm{mg}$ bid tofacitinib with a treat to target goal of Low Disease Activity (LDA) or remission depending on the Clinical Activity Index (CDAI) score at Baseline. If the target was not met and sustained for 3 months, the dose of tofacitinib was increased to $10 \mathrm{mg}$ bid in an attempt to reach target. MRIs of the hand/wrist were blindly read by a musculoskeletal radiologist using a rheumatoid arthritis MRI scoring system (RAMRIS). A CDAI score of $>10$ was needed at study entry.

Results: Of the 20 enrolled patients, 6 remained at $5 \mathrm{mg}$ bid and 14 were dose escalated to $10 \mathrm{mg}$ bid most at the 12 week period. Of the $5 \mathrm{mg}$ bid group, 3 completed the trial at target and 3 early termed (ET) for lack of efficacy, relocation and AE. Structurally, there was no change in erosions in all 3 patients; 2 showed regression of synovitis and 1 showed no change; 2 showed regression in osteitis and 1 no change. Of the 14 patients escalated to $10 \mathrm{mg}$ bid, 11 completed the trial with 7 remissions, 2 at LDA, and 1 at MDA. 3 patients ET due to lack of efficacy. In the $10 \mathrm{mg}$ bid group, 9 patients showed no change in erosions, 1 regression and 1 progression. 5 patients showed no change in synovitis and 6 showed regression, and 7 showed no change in osteitis, 3 showed regression and 1 showed progression. The CRP values correlated with the improvement of 